\title{
ThOUghts on Evidence-Based Medicine ANd InTERLAminar EPIDURAL INJECTIONS
}

To the Editor:

Recently, I was asked to co-author a review of the scientific evidence for, or against, the use of epidural steroid injections to treat pain. Although there have been many studies published over the years showing efficacy for interlaminar epidural injections, none met the criteria for our report because most were not randomized and none evaluated injections using fluoroscopy. On the other hand, recent studies of epidural injection have focused on the fluoroscopically-guided, transforaminal approach, and hence, high quality scientific articles evaluating the use of transforaminal epidural injections are available for review. After analysis of the extant scientific literature, our panel of experts concluded that there are randomized, controlled data to support the use of transforaminal epidural injections to treat lumbar radicular pain, but there are no randomized, controlled studies of fluoroscopically-guided interlaminar epidural injections to support or refute the use of this procedure (1).

Within two weeks of the publication of our review, a large health plan in our state informed pain physicians that they would no longer pay for interlaminar epidural injections and would only reimburse for transforaminal epidurals. The medical director of the plan cited our published review identifying lack of evidence to support interlaminar epidural injection as the basis for the health plan's decision. I responded to the health plan's policy with a letter discussing my views on evidencebased medicine in general, and epidural steroid injections in particular, and I would like to share my thoughts with the readers of Pain Physician journal.

\section{Art vs. science in medical practice}

The primary role of the practicing physician is to alleviate suffering and improve the health of patients. To provide effective medical care, the physician must combine his or her personal experience and knowledge of anatomy, pathophysiology, and pharmacology together with available scientific data in order to formulate a rational plan of care.

Regarding personal experience and knowledge, medicine is an art and a science and the art of medicine has to do with the cumulative experience, judgment, and wisdom amassed by physicians over time. Throughout the history of medicine, physicians have often relied on their seasoned judgment and experience to help alleviate suffering in their patients. While most physicians are eager to incorporate the findings of randomized, controlled studies into their medical practices, there are many situations where high quality scientific data are lacking. In these situations good physicians may rightly choose to use their understanding of anatomy, pathophysiology, and pharmacology, as well as their past experience and whatever less-than-perfect scientific data are available to provide medical treatment for their patients. In pain clinic practice, this reality is mirrored by the fact that virtually all major pain management textbooks, including Bonica's Management of Pain, Melzack and Wall's Textbook of Pain, Practical Management of Pain by Raj and Waldman's Interventional Pain Management describe many treatments that lack the support of randomized, controlled data. These textbooks, like medical textbooks in all fields of medicine, seek to summarize cumulative knowledge, including knowledge gained from case studies, common experience, and expert consensus, to help practicing physicians effectively treat their patients. Likewise, pain specialty organizations, such as the International Spinal Injection Society (ISIS) and the American Society of Interventional Pain Physicians (ASIPP) routinely teach interventional pain procedures which may lack the support of randomized, controlled data. These procedures are taught because they have a sound theoretical underpinning, they have the support of the experts within these organizations, and are generally accepted as effective treatments. To rely solely on randomized, controlled data to determine medical practice limits the ability of the physician to relieve suffering and, in certain circumstances, puts patients in jeopardy. In fact, if we provided only treatments that are supported by randomized controlled data we often would provide no treatment at all.

Regarding scientific data, while anecdote, case series, expert consensus, and non-randomized trials do not rise to the level of randomized, controlled research, this does not mean that studies which are not randomized and controlled are worthless. Results from less-than-perfect studies may be better than no studies at all, and should be considered along with other variables when assessing the effectiveness of a well-established medical practice or procedure. Furthermore, the absence of randomized, controlled studies to support well-established medical practices is not proof of lack of effectiveness for these practices. Lack of data for commonly-accepted treatments simply identifies the need for future scientific study. Conversely, when randomized, controlled data from more than one high quality scientific study show a lack of effect for a commonly accepted treatment, the procedure should be abandoned.

\section{The transition toward evidenced-based} medical practice

Most physicians support evidencebased medicine and believe that rigorous scientific investigation will lead to more effective medical care. But the transition from less scientific care to more scientific care will take time and it may be decades before physicians function in an environment where the majority of their treatments have scientifically-proven efficacy. If we were to suddenly stop using treatments that are not currently supported by randomized, controlled data we 
would needlessly abandon effective therapies, and do a disservice to the patients who have come to rely on these therapies. Abandoning medical treatments that have withstood the test of time and are widely practiced because randomized controlled trials have yet to be done, may cause unnecessary patient suffering.

\section{The case for interlaminar epidural steroids}

The purpose of most epidural steroid injections is to deposit steroid, usually mixed with local anesthetic, into the epidural space for therapeutic effect. The epidural space can be entered at various levels and by various techniques (interlaminar, transforaminal, caudal, via catheter) but the end result is essentially the same - deposition of steroid into the epidural space. Although transforaminal epidural injections may target the anterior epidural space and dorsal root ganglion more selectively, these structures can be effectively targeted using interlaminar injection approaches. Indeed, interlaminar epidurograms frequently document dispersal of medication into the anterior epidural space and onto one or more target spinal nerve roots. The key point for any epidural injection is that it be performed and confirmed with fluoroscopy, whichever approach is used. In other words, a technically adequate epidural steroid injection is a technically adequate epidural steroid injection and scientific data supporting the effectiveness of transforaminal epidural steroid injections provides evidence for the effectiveness of properly performed epidural steroid injections in general. There is nothing magical about the transforaminal route of administration, and it is reasonable to assume that any therapeutic effect of transforaminal steroid injection is caused by deposition of the steroid into the epidural space; depositing steroid onto the same structure using an interlaminar, caudal, or catheter technique will have a similar result.

In practice, most experienced spinal injectionists evaluate multiple variables including pain pattern, body habitus, individual spinal anatomy, and findings on MRI scan when deciding whether to perform an epidural injection via the interlaminar, caudal or transforaminal route. The interlaminar approach is often chosen over the transforaminal route because:

a. It is technically easier.

b. It is often less painful.

c. It is allows for wide dispersal of medication to treat multilevel and bilateral radicular pain patterns.

d. It carries an inherently lower risk of spinal cord injury because the segmental radiculo-medullary spinal feeding arteries variably present in neural foramina cannot be accessed from an interlaminar approach.

Although past studies regarding interlaminar epidural injection are not randomized and controlled and have generally evaluated injections performed without fluoroscopy, these studies do provide some element of scientific support for this procedure. Conversely, there are no sound scientific data that show a lack of effect for interlaminar epidural steroid injections that were done with fluoroscopic guidance. Most pain specialists would agree that definitive randomized and controlled studies should evaluate interlaminar epidural injections done with proper technique using fluoroscopic guidance. But to suddenly abandon the interlaminar method in favor of the transforaminal approach for every case does patients a disservice and puts them at increased risk.

In summary, the movement toward evidenced-based medical practice is pushing interventional pain management in a generally positive direction. However, the fact that health plans are using the guise of evidenced-based medicine to deny routine and well-established pain clinic procedures is a worrisome sign that portends increasing infringement on the pain physician's ability to effectively care for patients. But regardless of our concerns, the trend toward regulation and accountability in medical practice is unstoppable and moving forward at full speed. I applaud the efforts of ASIPP to provide scientific support for our specialty. Regarding epidural steroid injections, pain physicians should pro-actively generate the randomized and controlled data needed to support the fluoroscopically-guided interlaminar approach.

\section{Author AfFiliation: \\ David M. Schultz, MD \\ Medical Director \\ Medical Advanced Pain Specialists \\ 2104 Northdale Blvd NW, Suite 220 \\ Minneapolis, MN 55433 \\ E-mail:dschultz@painphysicians.com}

\section{RefERENCE}

1. Schultz DM, Schellhas $K$, Hurdle $M$. Fluoroscopically Guided Transforaminal Epidural Steroid Injections for Lumbar Radicular Back Pain, Institute for Clinical Systems Improvement: Technology Assessment Review, August 2004 